\title{
Severe Human Bocavirus 1 Respiratory Tract Infection in an Immunodeficient Child With Fatal Outcome
}

\section{Tabatabai, Julia}

2019-09

Tabatabai , J , Fakhiri , J , Meyburg , J , Linse , K-P , Xu , M , Söderlund-Venermo , M , Grimm , D \& Schnitzler , P 2019 , ' Severe Human Bocavirus 1 Respiratory Tract Infection in an Immunodeficient Child With Fatal Outcome ' , Pediatric Infectious Disease Journal , vol. 38 , no. 9 , pp. e219-e222 . https://doi.org/10.1097/INF.0000000000002354

http://hdl.handle.net/10138/323358

https://doi.org/10.1097/INF.0000000000002354

cc_by_nc

acceptedVersion

Downloaded from Helda, University of Helsinki institutional repository.

This is an electronic reprint of the original article.

This reprint may differ from the original in pagination and typographic detail.

Please cite the original version. 
1 Severe human bocavirus 1 respiratory tract infection in an

\section{2 immunodeficient child with fatal outcome}

3 Julia Tabatabai, MD ${ }^{\text {a,b,c }}$, Julia Fakhiri ${ }^{\text {a,d }}$, Jochen Meyburg, MD ${ }^{\mathrm{b}}$, Kai-Philipp Linse ${ }^{\mathrm{a}, \mathrm{c}, \mathrm{d}}$, Man

$4 \mathrm{Xu}^{\mathrm{e}}$, Maria Söderlund-Venermo, $\mathrm{PhD}^{\mathrm{e}}$, Dirk Grimm, $\mathrm{PhD}^{\mathrm{a}, \mathrm{c}, \mathrm{d}, \mathrm{f}}$, Paul Schnitzler, $\mathrm{PhD}^{\mathrm{a}}$

6 Center for Infectious Diseases ${ }^{\mathrm{a}}$, Center for Childhood and Adolescent Medicine ${ }^{\mathrm{b}}$, University

7 Hospital Heidelberg, Heidelberg, Germany; German Center for Infection Research (DZIF),

8 partner site Heidelberg, Germany ${ }^{\mathrm{c}}$; BioQuant Center, University of Heidelberg, Heidelberg,

9 Germany $^{\text {d; }}$ Department of Virology, University of Helsinki, Helsinki, Finland ${ }^{\text {; }}$, Cluster of

10 Excellence CellNetworks, Heidelberg, Germany ${ }^{\mathrm{f}}$

\section{Corresponding author}

13 Dr. med. Julia Tabatabai

14 E-mail address: julia.tabatabai@med.uni-heidelberg.de

Keywords:

parvovirus, bocavirus, respiratory tract infection, immunodeficiency, viral shedding

Short title: Fatal bocavirus-1 infection in an immundeficient child

Running head: Fatal bocavirus-1 infection 
We report a case of lower respiratory tract infection with human bocavirus 1 (HBoV1) in an immunodeficient 6-month-old boy leading to respiratory failure with fatal outcome. PCR of

\section{Abstract}

serum/tracheal secretions revealed exceptionally high HBoV1-DNA levels and immunoassays showed seroconversion indicating an acute primary HBoV1 infection. All assays for other pathogens were negative, strongly suggesting that $\mathrm{HBoV} 1$ was the causative agent in this case.

\section{Background}

Human bocavirus $(\mathrm{HBoV}) 1$ is a recently identified viral agent that belongs to the family of Parvoviridae and comprises a non-enveloped capsid with a linear single-stranded DNA genome [1]. Viral DNA has been detected mainly in nasopharyngeal secretions, and in serum or blood samples of younger children with upper or lower respiratory tract infections (RTI). Besides HBoV1, which is predominantly detected in the respiratory tract, three other bocaviruses, HBoV2 -4 , are mainly found in stool.

Detection of HBoV1 DNA in airway samples of children with RTI is frequently combined with other viruses or bacteria because HBoV1 persists and is shed for a prolonged period. This makes interpretation of a PCR-positive test result difficult. The high detection rate of multiple respiratory viruses in an airway specimen, and the presence of HBoV1 DNA in asymptomatic children, thereby complicate the diagnosis of acute HBoV1 infections [2]. In addition to detection of HBoV1 DNA in airway samples, other diagnostic methods, such as serology, should be used. With accurate diagnostic methods, acute HBoV1 infection has been shown to cause mild to life-threatening RTIs. Due to the lack of an animal model, the Koch's postulates have not been formally fulfilled, thus proving clinical relevance is challenging. 
Life-threatening infections are rare. Here we present a case of HBoV1 lower RTI, diagnosed by both PCR and serology, leading to severe respiratory failure with fatal outcome in an immunodeficient child.

\section{Case presentation}

A six-month-old boy, the first child of consanguineous parents, was transferred from Dubai, United Arab Emirates, to the University Hospital Heidelberg, Germany, for further diagnostic workup of an unspecified syndrome including failure to thrive, distinct psychomotor retardation, multiple osseous malformations, microcephaly due to cerebral atrophy, blindness, and symptoms of an acute hemolytic uremic syndrome. Shortly after admission, a T-cell defect was diagnosed and whole-exon screening identified a homozygous mutation in the NIN gene which codes for Ninein, a protein crucial for mitosis. The NIN gene mutation was regarded as the cause of the complex syndrome. The cause of the immunodeficiency found in our patient was an impaired cytokine response in combination with insufficient formation of antigen-presenting cells to $\mathrm{T}$ lymphocyte synapses leading to a functional T-cell deficiency. Because of feeding difficulties and significant dysphagia, a percutaneous gastrostomy tube as well as a Hickman line were placed. The hemolytic uremic syndrome was treated with eculizumab; however, proteinuria and oliguria persisted and required high doses of diuretics. About ten days after admission, the boy developed pneumonia and was transferred to the intensive care unit where he had to be intubated, and was mechanically ventilated. He developed an acute respiratory distress syndrome (ARDS) with multiple critical deteriorations. One week after admission, an initial radiograph was performed in the context of the surgery for a Hickman line and a gastrostomy tube. He did not show signs of a preceding lung injury (Fig. 1). Mechanical ventilation was difficult with an $\mathrm{FiO}_{2}$ of 1.0, high inspiratory pressures up to 40/11 mbar, and intermittent use of high frequency oscillation and 
inhaled nitric oxide. Tracheostomy was performed after six weeks of mechanical ventilation. Because of atelectasis, pulmonary secretion, and bronchospasm, the mechanical ventilation had to be intensified several times. Elevated CRP values (average $92.4 \mathrm{mg} / 1$, peak $221.7 \mathrm{mg} / \mathrm{l}$, normal $<5 \mathrm{mg} / \mathrm{l})$ were first noted one week prior to intubation and mechanical ventilation, and did not normalize over the next four months. This prompted antibiotic treatment with different combinations of cefotaxime, meropenem, ciprofloxacin, teicoplanin, linezolid, and erythromycin with no clinical effect. Apart from Staphylococcus epidermidis detected in a single blood culture, bacterial as well as fungal cultures and PCR for atypical microorganisms in tracheal secretions were always negative. Tracheal secretions were also tested in a real time multiplex respiratory PCR (Fast Track Diagnostics respiratory pathogens 21, Luxembourg) for influenza A virus including H1N1, influenza B virus, rhinovirus, respiratory syncytial virus, bocavirus, adenovirus, parainfluenza virus 1 through 4, four coronaviruses (NL63, 229E, OC43, HKU1), parechovirus, enterovirus, human metapneumovirus $\mathrm{A} / \mathrm{B}$ and Mycoplasma pneumoniae. Additionally, these samples were also tested for herpes simplex virus and varicella zoster virus DNA by in-house PCR and for bacteria and fungi by culture. Human bocavirus $1(\mathrm{HBoV} 1)$ was the only pathogen detected in tracheal secretions. All serial tracheal secretions were positive for HBoV1 DNA, which was present in high copy numbers in some samples (Fig. 2A). The highest viral load in respiratory samples of $3.1 \times 10^{9}$ copies $/ \mathrm{ml}$ was detected about day 20 of hospitalisation, one week after pneumonia was diagnosed and mechanical ventilation initiated. The viral load decreased slowly in respiratory samples but DNA was detectable for several months. Blood was taken from the patient once per week and HBoV1 DNA was detectable in serum for 50 days with a peak of $2.0 \times 10^{3}$ copies $/ \mathrm{ml}$. The specificity of the real-time PCR was confirmed with a qPCR and an in-house PCR followed by DNA sequence analysis of the amplified product. The qPCR assays were performed using the 1xSensiMix SYBR No-ROX Kit (Bioline Reagents Ltd, London, UK) 
with HBoV1-specific primers (forward primer 5'-CCTATATAACCTGCTGCACTTCCT-3', reverse primer 5'-AAGCCATAGTAGACTCACCACAAG-3').

The complete VP1 gene (2016 bp) of the HBoV1 genome was amplified by an in-house PCR assay including the HBoV1-specific primers (forward primer 5'GTTACGTCTCGAAGATTACAACACTTTATTGATGTTTG-3', reverse primer 5'GTTACGTCTCAGCAGATGCCTCCAATTAAGAGACA-3'). The PCR product was purified and subsequently sequenced. The sequence (accession no. MG680946) was then aligned with different $\mathrm{HBoV}$ strains reported in GenBank and subjected to a phylogenetic analysis (Fig. 3). This confirmed a 99\% identity of the study sample with HBoV1, thus verifying the specificity of the multiplex respiratory PCR, the qPCR and the immunoassays. Moreover, the phylogenetic analysis revealed a close relationship to a previously reported HBoV1 isolate from Egypt [3] (GB accession no. KU557404.1, as shown in Fig. 3). By following the course of infection over a period of four months, we detected the emergence and persistence of a mutation at amino acid position 590 (VP1 numbering) that results in an amino-acid change from threonine to serine.

HBoV1-specific IgG and IgM were measured by highly sensitive and specific competition immunoassays based on HBoV1-like particles [4, 5]. Both IgG and IgM antibodies against HBoV1 were detected and seroconversion was observed (Fig. 2B), indicating an acute HBoV1 infection. Thus, HBoV1 was considered the most likely cause of ARDS. The patient died of multi-organ failure following four months of mechanical ventilation.

\section{Discussion and conclusions}

Human bocavirus 1 (HBoV1) was discovered in 2005 by Allander et al. in respiratory secretions [1] and is increasingly recognized as a cause of pediatric respiratory tract infections 
worldwide $[2,6,7]$. By PCR of airway samples, HBoV1 DNA has been detected in $2-20 \%$ of children with respiratory tract infection, whereas $40-75 \%$ of the HBoV1 DNA-positive patients show co-detections with other respiratory pathogens $[8,9]$.

However, it is important to acknowledge that almost all routine testing and published studies of HBoV1 infections rely on only PCR testing of respiratory secretions. HBoV1 DNA can by sensitive PCRs be detected for months or even up to a year after acute infection, leading to co-detections and false clinical diagnoses — and thereby, inaccurate disease associations [10 13]. Mere qualitative PCR is therefore not an adequate method for diagnosing acute $\mathrm{HBoV} 1$ infections, instead a combination of other diagnostic means including qPCR of respiratory samples and serum, as well as serology should be applied $[4,14]$.

By utilizing accurate diagnostics, increasing evidence has been gathered of HBoV1 being the cause of mild to severe upper and lower respiratory tract infections in children over 6 months of age $[6,7]$. HBoV1 may cause also life-threatening complications of lower respiratory tract infection including emphysema, pneumomediastinum, pneumothorax and acute respiratory failure [15 - 18]. In addition, both Sadeghi et al. [19] and Krakau et al. [20] described immunocompromised adult patients suffering from an advanced myelodysplastic syndrome with severe HBoV1 pneumonia with fatal outcome. Further fatal cases associated with HBoV1 infection were described in an adult and a pediatric patient with underlying lung diseases [21, 22]. The need for ventilator support for four months reflecting the extensive lung damage is perfectly explained by the severe ARDS leading to lung fibrosis, impaired gas exchange and eventually death of the patient. Most of the damage to the lungs of patients that do not recover from ARDS is caused by pulmonary inflammation and interstitial fibrosis. It has been shown that $\mathrm{HBoV} 1$ infection of in vitro airway epithelium cultures inhibits apoptosis and induces pyroptotic cell death, resulting in tissue injury and inflammation [23, 24]. Persistent HBoV1 infection of the lungs in immunocompromised children may thus lead to 
lung tissue injury. It can therefore be hypothesized that persistent $\mathrm{HBoV} 1$ infection in this child directly damaged the alveolar tissue.

Nevertheless, the significance of HBoV1 infection as a cause of death, as described in this case of an immunodeficient child, is not easy to determine. However, viral DNA of exceptionally high copy numbers of $5 \times 10^{9}$ copies/ml was observed in tracheal secretions at the same time as it occurred in serum, pointing to an acute HBoV1 infection. In general, acute HBoV1 infection is accompanied by the presence of viral DNA in serum [4]. After atypical hemolytic-uremic syndrome had been diagnosed at the age of three months, eculizumab was given five times with approximately three weeks between each application. The last dose was applied 18 days before the respiratory decompensation. It is tempting to speculate that the very high viral load reflects the unusually complicated clinical course and the immunocompromised status of the patient. High viral loads of HBoV1 are associated with respiratory symptoms whereas low viral loads mostly indicate longitudinal asymptomatic shedding $[2,4,6,14,25,26]$. Detection of HBoV1 DNA in serum has further been more tightly linked to symptoms than DNA in respiratory samples. The functional T-cell defect in our case is regularly found in Schimke immuno-osseous dysplasia that was initially suspected but ruled out genetically. Other cases with severe HBoV1 infection in patients with T-cell defect or immunodeficiency have been reported previously [10, 19, 27, 28].

High HBoV1 DNA copy numbers in airway samples, short viremic phase, detection of HBoV1-specific IgM and seroconversion of IgG antibodies have been shown to be accurate diagnostic markers in children with acute $\mathrm{HBoV} 1$-induced respiratory illness and can thereby separate acute infection from asymptomatic virus shedding [4]. The usefulness of the applied serology has been documented in studies of children with acute wheezing or with communityaquired pneumonia $[4,29,30]$. HBoV1 IgM positivity correlates with both HBoV1 viremia and seroconversion of $\operatorname{IgG}$ in paired serum samples, whereas healthy subjects are generally 
IgM negative with stable IgG absorbance levels [4]. While in this case, multiplex PCR for 19 other respiratory viruses and mycoplasma was negative, the course of the disease perfectly matched a primary infection by HBoV1, supported by serology and detection of HBoV1 DNA in serum.

The emergence and persistence of a mutation at amino acid position 590 that resulted in an amino-acid change from threonine to serine reflects either (i) a mixed primary infection with two HBoV1 variants, where one dominates over time, (ii) a secondary infection with a different HBoV1 variant, as hypothesized in Martin et al. [12], or (iii) the occurrence of a de novo mutation that fostered clonal selection. Further time-course studies with more patients and the use of deep sequencing approaches are required to unanimously resolve these possibilities.

This report illustrates that blood sampling is important for linking HBoV1 with disease, and it indicates that $\mathrm{HBoV} 1$ should be considered in severe respiratory tract disease in children. $\mathrm{HBoV} 1$ is the most probable cause of respiratory tract disease if the patient has a high viral load in respiratory samples accompanied by viremia, if $\mathrm{HBoV} 1$ is the only pathogen detected, and if an acute primary HBoV1 infection is diagnosed by serological testing $[4,8]$. We detected HBoV1 DNA in both respiratory and serum samples. Moreover, the serologic results indicate that this child had an acute primary HBoV1 infection. The dramatic increase of HBoV1 load in tracheal secretions and viral dissemination most likely resulted from a progressive impairment of cellular immunity. The observation that all other viral and microbiological assays were negative, strongly suggest that $\mathrm{HBoV} 1$ was the causative agent of respiratory failure and death in the present case.

\section{Acknowledgements}


194 The authors would like to thank all technicians in the virology diagnostic laboratory for excellent technical support.

\section{Funding}

This study was funded in part by the Cystic Fibrosis Foundation (CFF; grant GRIMM15XX0) for J.F., K.-P.L. and D.G. K.-P.L. was supported by an MD stipend from the German Center for Infection Research (DZIF; BMBF). J.T. received a clinical leave stipend from DZIF. M.X. was funded by the China Scholarship Council and M.S-V. by the Sigrid Jusélius Foundation and the Life and Health Medical Association.

\section{Availability of data and materials}

The data supporting the conclusions of this article are included within this article. The sequence generated and analysed for this report is available in GenBank under the accession number MG680946.

\section{Author's contributions}

Study design: JT, PS; Clinical evaluation: JT, JM, PS; Laboratory testing: JF, K-PL, MX; Data analysis and manuscript preparation: JT, JF, K-PL, MS-V, DG, PS. All authors reviewed and approved the final manuscript.

\section{Ethics approval}

The study was approved by the Ethical Research Board of the University Hospital Heidelberg, Germany (S-547/2015). All samples and medical information included in this study were obtained during routine medical care.

\section{Competing interests}

The authors declare that they have no competing interests. 


\section{References}

1. Allander T, Martti TT, Eriksson M, Bjerkner A, Tiveljung-Lindell A, Andersson B. Cloning of a human parvovirus by molecular screening of respiratory tract samples. Proc Natl Acad Sci USA 2005, 102:12891-96.

2. Broccolo F, Falcone V, Esposito S, Toniolo A. Human bocaviruses: possible etiologic role in respiratory infection. J Clin Virol 2015, 72:75-81.

3. Abdel-Moneim AS, Kamel MM, Hassan NM. Evolutionary and genetic analysis of human bocavirus genotype-1 strains reveals an evidence of intragenomic recombination. J Med Microbiol 2017, 66:245-54.

4. Söderlund-Venermo M, Lahtinen A, Jartti T, Hedman L, Kemppainen K, Lehtinen P, Allander T, Ruuskanen O, Hedman K. Clinical assessment and improved diagnosis of bocavirus-induced wheezing in children, Finland. Emerg Infect Dis 2009, 15:1423-30.

5. Kantola K, Hedman L, Allander T, Jartti T, Lehtinen P, Ruuskanen O, Hedman K, Söderlund-Venermo M. Serodiagnosis of human bocavirus infection. Clin Infect Dis 2008, 46:540-6.

6. Jartti T, Hedman K, Jartti L, Ruuskanen O, Allander T, Söderlund-Venermo M. Human bocavirus-the first 5 years. Rev Med Virol 2012, 22:46-64.

7. Qiu J, Söderlund-Venermo M, Young NS. Human Parvoviruses. Clin Microbiol Rev 2017, 30:43-113.

8. Christensen A, Nordbø SA, Krokstad S, Wesenberg Rognlien AG, Døllner H. Human bocavirus in children: mono-detection, high viral load and viraemia are associated with respiratory tract infection. J Clin Virol 2010, 49:158-62. 
9. Weissbrich B, Neske F, Schubert J, Tollmann F, Blath K, Blessing K, Kreth HW. 2006. Frequent detection of bocavirus DNA in German children with respiratory tract infection. BMC Infect. Dis. 6:109.

10. Koskenvuo M, Möttönen M, Waris M, Allander T, Salmi TT, Ruuskanen O. Human bocavirus in children with acute lymphoblastic leukemia. Eur J Pediatr 2008, 167:1011-5.

11. Blessing K, Neske F, Herre U, Kreth H-W, Weissbrich B. Prolonged detection of human bocavirus DNA in nasopharyngeal aspirates of children with respiratory tract disease. Ped Infect Dis J 2009, 28:1018-9.

12. Martin ET, Kuypers J, McRoberts JP, Englund JA, Zerr DM. Human bocavirus 1 primary infection and shedding in infants. J Infect Dis 2015, 212:516-24.

13. Schildgen O Müller A, Allander T, Mackay IM, Völz S, Kupfer B, Simon A. Human bocavirus: passenger or pathogen in acute respiratory tract infections? Clin Microbiol Rev 2008, 21:291-304.

14. Xu M, Arku B, Jartti T, Koskinen J, Peltola V, Hedman K, Söderlund-Venermo M. 2017. Comparative Diagnosis of Human Bocavirus 1 Respiratory Infection With Messenger RNA Reverse-Transcription Polymerase Chain Reaction (PCR), DNA Quantitative PCR, and Serology. J. Infect. Dis. 215:1551-7.

15. Edner N, Castillo-Rodas P, Falk L, Hedman K, Söderlund-Venermo M, Allander T. Life-threatening respiratory tract disease with human bocavirus-1 infection in a 4year-old child. J Clin Microbiol 2012, 50:531-2.

16. Körner RW, Söderlund-Venermo M, van Koningsbruggen-Rietschel, Kaiser R, Malecki M, Schildgen O. Severe human bocavirus infection, Germany. Emerg Infect Dis 2011, 17:2303-5. 
17. Ursic T, Steyer A, Kopriva S, Kalan G, Krivec U, Petrovec M. Human bocavirus as the cause of a life-threatening infection. J Clin Microbiol 2011, 49:1179-81.

18. Eskola V, Xu M, Söderlund-Venermo M. Severe lower respiratory tract infection caused by human bocavirus 1 in an infant. Pediatr Infect Dis 2017, 36:1107-8.

19. Sadeghi M, Kantola K, Finnegan DPJ, McCaughey C, Hedman L, SöderlundVenermo M, Hedman K. Possible involvement of human bocavirus 1 in the death of a middle-aged immunosuppressed patient. J Clin Microbiol 2013, 51:3461-3.

20. Krakau M, Brockmann M, Titius B, Limmroth C. Khalfaoui S, Schildgen V, Dormann A, Schildgen O. Acute human bocavirus infection in MDS patient, Cologne, Germany. J Clin Virol 2015, 69:44-7.

21. Ursic T, Krivec U, Kalan G, Petrovec M. Fatal human bocavirus infection in an 18month-old child with chronic lung disease of prematurity. Pediatr Infect Dis J 2015, $34: 111-2$.

22. Dieninghoff D, Karagiannidis C, Straßmann S, Pieper M, Dammaschek S, Zabner J, Klingelhutz A, Windisch W, Brockmann M, Schildgen O, Schildgen V. Fatal HBoV-1 infection in adult female cystic fibrosis patient. Hum Path Case Rep 2017, 7:51-2.

23. Deng, X, Zou, W, Xiong, M, Engelhardt JF, Ye SQ, Yan Z, Qiu J. Human parvovirus infection of human airway epithelia induces pyroptotic cell death by inhibiting apoptosis. J Virol 2017, 91: e01533-17.

24. Huang, Q, Deng, X, Yan, Z, Cheng F, Luo Y, Shen W, Lei-Butters DC, Chen AY, Li Y, Tang L, Söderlund-Venermo M, Engelhardt JF, Qiu J. Establishment of a reverse genetics system for studying human bocavirus in human airway epithelia. PLoS Pathog 2012, 8: e1002899. 
25. Allander T, Jartti T, Gupta S, Niesters HGM, Lehtinen P, Österback R, Vuorinen T, Waris M, Bjerkner A, Tiveljung-Lindell A, van den Hoogen BG, Hyypiä T, Ruuskanen O. Human bocavirus and acute wheezing in children. Clin Infect Dis 2007, 44:904-10.

26. Jacques J, Moret H, Renois F, Lévêque N, Motte J, Andréoletti L. Human bocavirus quantitative DNA detection in French children hospitalized for acute bronchiolitis. J Clin Virol 2008, 43:142-7.

27. Schenk T, Strahm B, Kontny U, Hufnagel M, Neumann-Haefelin D, Falcone V. Disseminated bocavirus infection after stem cell transplant. Emerg Infect Dis 2007, $13: 1425-7$

28. Müller A, Klinkenberg D, Vehreschild J, Cornely O, Tillmann RL, Franzen C, Simon A, Schildgen O. Low prevalence of human metapneumovirus and human bocavirus in adult immunocompromised high risk patients suspected to suffer from Pneumocystis pneumonia. J Infect 58:227-31.

29. Don M, Söderlund-Venermo M, Valent F, Lahtinen A, Hedman L, Canciani M, Hedman K, Korppi M. Serologically verified human bocavirus pneumonia in children. Ped Pulm 2010, 45:120-6.

30. Nascimento-Carvalho CM, Cardoso MRA, Meriluoto M, Kemppainen K, Kantola K, Ruuskanen O, Hedman K, Söderlund-Venermo M. Human bocavirus infection diagnosed serologically among children admitted to hospital with community-acquired pneumonia in a tropical region. J Med Virol 2012, 84:253-8. 
Fig. 1.

310 Chest X-ray showing bilateral opacities as sign of acute respiraotory distress syndrome 311 (ARDS)

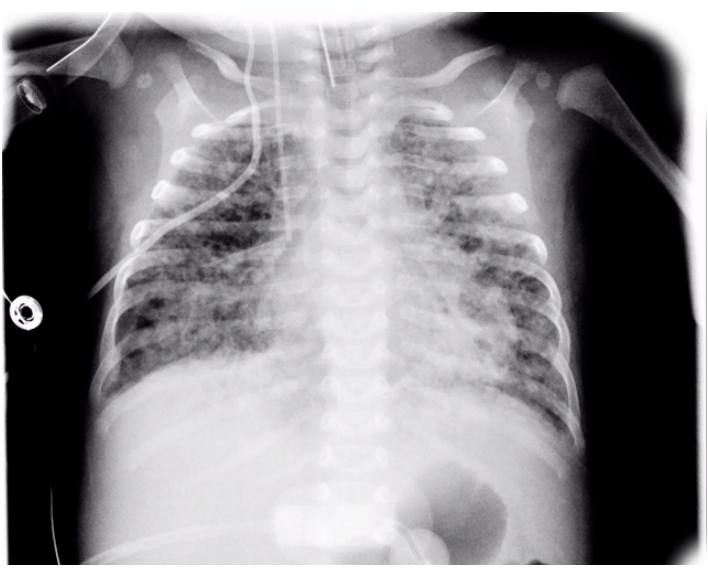

Fig. 2.

HBoV1 diagnostic findings. A) Detection of HBoV1 DNA in tracheal secretions and serum. onset.

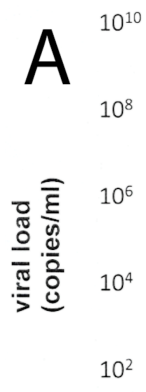

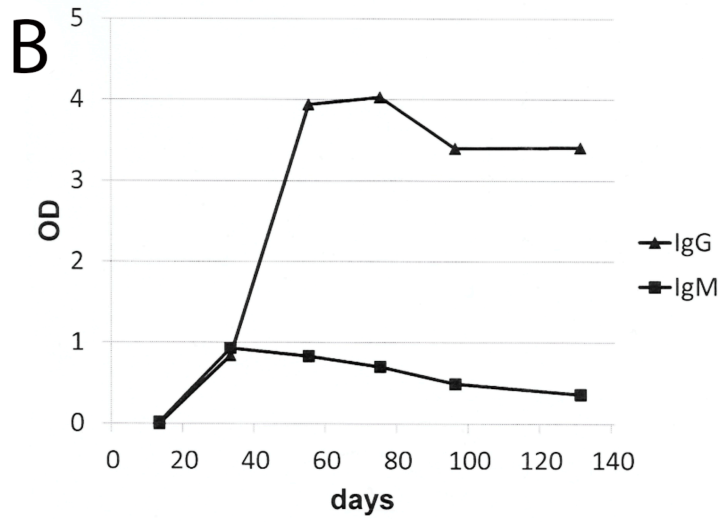


Fig. 3.

323 Maximum likelihood phylogenetic consensus tree for the VP ORF nucleotide sequence of the 324 HBoV1 isolate (V1445149) studied here. The numbers next to the nodes indicate the value of 500 bootstrap analyses. To root the tree, an outgroup of the indicated closely related members of the genus Bocaparvovirus was defined. Only bootstrap values higher than $50 \%$ are presented. Codon positions included were $1 \mathrm{st}+2 \mathrm{nd}+3 \mathrm{rd}+$ noncoding. Evolutionary analyses were conducted in MEGA 7.0.26.

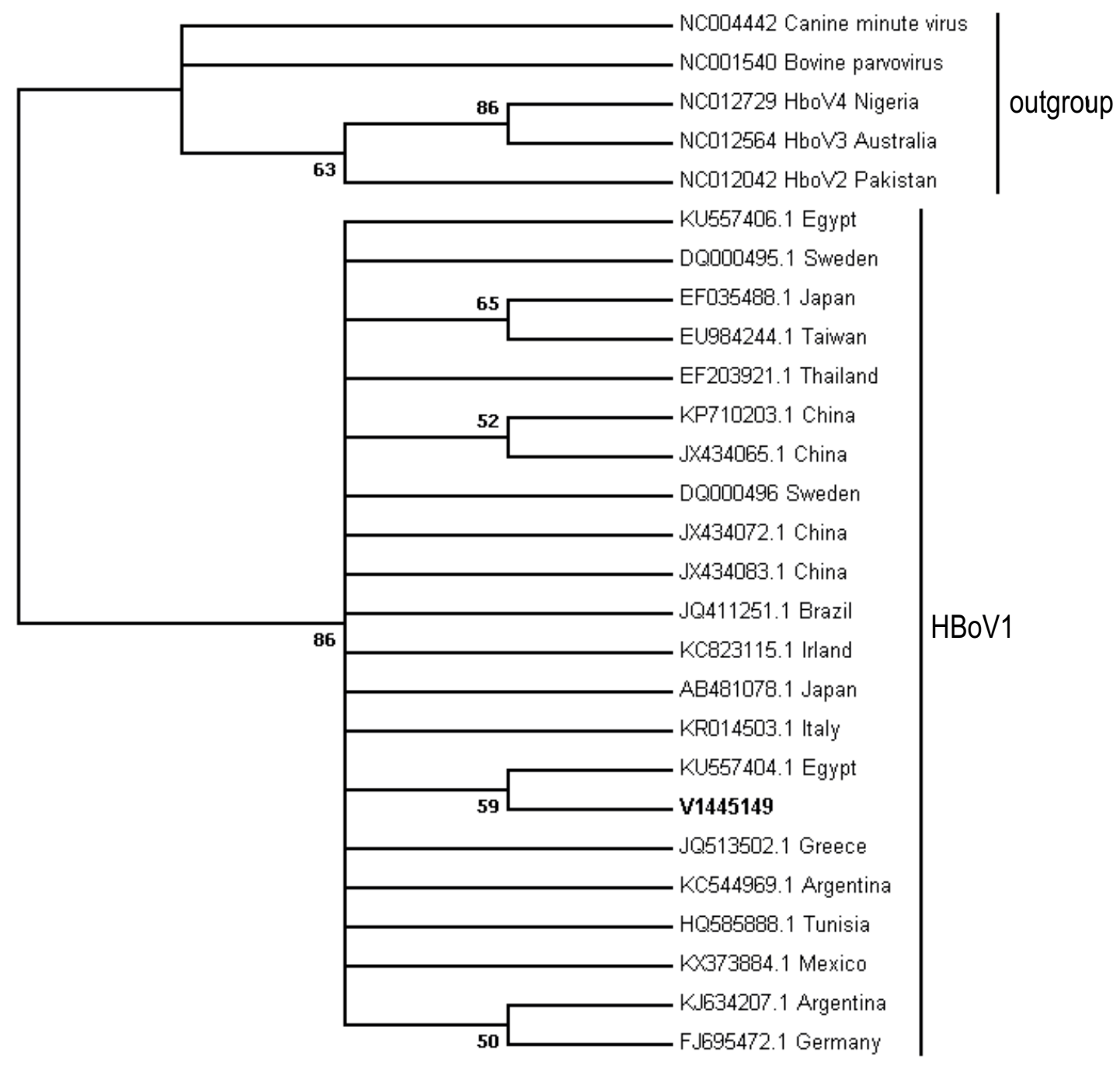

